\title{
Diboson Higgs and EWK measurements and triple gauge couplings with ATLAS and CMS
}

\author{
Protopapadaki Eftychia Sofia \\ (On behalf of the ATLAS and CMS Collaborations)* \\ CEA Saclay \\ E-mail: eftychia-sofia.protopapadaki@cern.ch
}

\begin{abstract}
This paper presents an overview of diboson production in pp collisions at 7 and $8 \mathrm{TeV}$ centerof-mass energy based on data recorded by the ATLAS and CMS detectors at the LHC in 2011 and 2012. This includes cross section measurements of $\mathrm{W}$ and $\mathrm{Z}$ production in association with a photon and of WW, WZ and ZZ productions at the LHC. Using these final states, we set limits on anomalous triple gauge boson couplings. Higgs measurements in $\mathrm{ZZ}$ and WW decay modes are also presented. The leptonic decay modes of the $\mathrm{W}$ and $\mathrm{Z}$ bosons are used. Studies using the semi-leptonic decay modes of WW+WZ and WZ and ZZ final state are also shown.
\end{abstract}

XXII. International Workshop on Deep-Inelastic Scattering and Related Subjects, 28 April - 2 May 2014

Warsaw, Poland

\footnotetext{
* Speaker.
} 


\section{Introduction}

The diboson production at the LHC is very interesting to study, as dibosons can be produced either directly via the $t$ and $\mathrm{u}$-channels or indirectly from Higgs decays. Therefore, the electroweak sector can be precisely probed using dibosons and any deviation of the measured cross section from the standard model (SM) prediction will indicate new physics. Furthermore, the precise knowledge of the SM diboson cross section will permit a better Higgs study, as they consist an irreducible background to it. Finally, the study of the triple gauge boson couplings (s-channel), will also permit to search for new physics. In this document results obtained by both ATLAS [1] and CMS [2] colaborations are presented.

\section{Diboson cross sections}

$V \gamma$ The $V \gamma$ process, where $\mathrm{V}$ is a $\mathrm{Z}$ or $\mathrm{W}$ boson, is the one with the higher cross section among the diboson processes. This process has been studied at a center of mass energy $(\sqrt{(s)})$ of $7 \mathrm{TeV}$, with both ATLAS and CMS detectors, using 4.6 and $5 \mathrm{fb}^{-1}$, respectively. Three final states have been considered, one for the $\mathrm{W}$, when it decays in lepton ( $e$ or $\mu)$ - neutrino pair, and two for the $\mathrm{Z}$, when it decays in a lepton ( $e$ or $\mu$ ) or a neutrino pair. Thus the experimental signature is one high energy isolated photon produced in association with 0,1 or 2 high energy isolated leptons, and missing transverse energy in the first and third case, to account for the neutrinos. The main background for these processes are the $V+$ jets and the $\gamma+$ jets production. The cross section measurement for each final state was performed in a different fiducial volume. CMS cross section measurement for the $W \gamma \rightarrow l v \gamma$ channel is $37.0 \pm 0.8$ (stat.) \pm 4.0 (syst.) \pm 0.8 (lumi.) pb, to be compared to the NLO MCFM prediction $31.8 \pm 1.8 \mathrm{pb}$ [3]. For the same process, but for a much smaller fiducial volume, ATLAS finds $2.77 \pm 0.03$ (stat.) \pm 0.33 (syst.) \pm 0.14 (lumi.) pb, to be compared with $1.96 \pm 0.17 \mathrm{pb}$ prediction [4]. The two experiments see discrepancies with respect to the SM cross section of 1 and $2 \sigma$, respectively. The measurement for the $Z \gamma \rightarrow l l \gamma$ channel by CMS is $5.33 \pm 0.08$ (stat.) \pm 0.25 (syst.) \pm 0.12 (lumi.) pb and it is in agreement with the theory prediction $5.45 \pm 0.27 \mathrm{pb}$ [3]. Likewise, ATLAS measurement $1.31 \pm 0.02$ (stat.) \pm 0.11 (syst.) \pm 0.05 (lumi.) $\mathrm{pb}$ is in agreement with theory $1.18 \pm 0.05 \mathrm{pb}$ [4]. Finally, the measurement of the $Z \gamma \rightarrow v v \gamma$ process is also in agreement with theory, for both CMS, $0.0211 \pm 0.0042$ (stat.) \pm 0.0043 (syst.) \pm 0.0005 (lumi.) pb to be compared to $0.0219 \pm 0.0011 \mathrm{pb}$ [5], and ATLAS, $0.133 \pm 0.013$ (stat.) \pm 0.02 (syst.) \pm 0.005 (lumi.) pb to be compared to $0.156 \pm 0.012 \mathrm{pb}$ [4], since in both cases the large measurement uncertainties cover the measurement - prediction difference. It should be noted that newly computed NNLO QCD corrections on the $W \gamma$ and $Z \gamma$ processes for the experiments fiducial volumes, increase the predicted cross section by $20 \%$ and $6 \%$, respectively. Thus, this brings the $W \gamma$ measurement in line with the theory prediction $[6,7]$.

$W W \quad$ The opposite sign $W W$ study was performed with both CMS and ATLAS detectors at $\sqrt{(s)}$ of $7 \mathrm{TeV}$ with 4.9 and $4.6 \mathrm{fb}^{-1}$, respectively. CMS has also provided results using part of the $8 \mathrm{TeV}$ data, corresponding to an integrated luminosity of $3.5 \mathrm{fb}^{-1}$. The total $W W$ cross section was measured studying the leptonic final state, where both $\mathrm{W}$ decay to a lepton ( $e$ or $\mu)$ - neutrino pair. This final state has two prompt leptons and missing transverse energy due to the two neutrinos. The main background processes are the $W+$ jets, Drell-Yan and $t \bar{t}$ production. For the $7 \mathrm{TeV}$ 
analysis, ATLAS measured 51.9 \pm 2.0 (stat. $) \pm 3.9$ (syst. $) \pm 2.0$ (lumi.) pb [8] and CMS measured $52.4 \pm 2.0$ (stat.) \pm 4.5 (syst.) \pm 1.2 (lumi.) pb [9]. These two measurements are in good agreement between them, but both are up to $2 \sigma$ larger than the theory prediction $44.7 \pm 2.0 \mathrm{pb}$ and $47.0 \pm 2.0$ $\mathrm{pb}$, respectively. The two theory predictions are slightly different (covered by theory uncertainties) due to different PDF used: CT10 for ATLAS, MSTW for CMS. For the CMS $8 \mathrm{TeV}$ measurement the cross section is $69.9 \pm 2.2$ (stat.) \pm 5.6 (syst.) \pm 3.1 (lumi.) pb, which is up to two $\sigma$ away from the theory prediction $57.3_{-1.6}^{+2.3} \mathrm{pb}[10]$.

$W Z \quad$ The total $W Z$ cross section was measured by both experiments, using the 7 and $8 \mathrm{TeV}$ data. The signature of this process is three high energy isolated leptons, produced in association with missing transverse energy due to the neutrino. The main backgrounds are those containing fake leptons, such as $Z / W+j e t / \gamma$ and $t \bar{t}$, and the $Z Z$, when one of the four leptons falls outside the detector acceptance. The $7 \mathrm{TeV}$ measurement for ATLAS is $19.0_{-1.3}^{+1.4}$ (stat.) \pm 0.9 (syst. $) \pm 0.4$ (lumi.) pb [11] and for CMS is $20.76 \pm 1.32$ (stat.) \pm 1.13 (syst.) \pm 0.46 (lumi.) pb [12]. These numbers are within $1 \sigma$ away from the NLO MCFM+CT10 prediction $17.6_{-1.0}^{+1.1} \mathrm{pb}$. For the $8 \mathrm{TeV}$ data the ATLAS measurement is $20.3_{-0.7}^{+0.8}(\text { stat } .)_{-1.1}^{+1.2}(\text { syst } .)_{-0.6}^{+0.7}($ lumi. $)$ pb [13], which is in agreement with the NLO MCFM+CT10 prediction $20.3 \pm 0.8 \mathrm{pb}$, while CMS measures $24.61 \pm 0.76$ (stat. $) \pm$ 1.13 (syst.) \pm 1.08 (lumi.) pb [12], and thus sees an excess slightly higher than $1 \sigma$. In the CMS paper, the ratio of the $W^{+} Z$ cross section over the $W^{-} Z$ cross section has also been measured, and it is found to be $1.94 \pm 0.25$ (stat.) \pm 0.04 (syst.) for $7 \mathrm{TeV}$, and $1.81 \pm 0.12$ (stat.) \pm 0.03 (syst.) for $8 \mathrm{TeV}$. Both results are in agreement with the theory prediction.

$Z Z \quad T$ The $Z Z$ is the diboson process with the cleanest final state, as in the leptonic channel the signature is four high energy isolated leptons. Thus, the background is very low, and the systematics associated to this measurement are small. However, this diboson process is the one with the lower cross section, and hence, the analysis strategy is to maximize the acceptance. The total $\mathrm{ZZ}$ on shell cross section has been measured by both experiments using both 7 and $8 \mathrm{TeV}$ data. In addition to the four leptons final state, the measurement was also performed in the two leptonstwo neutrinos channel. This channel has high background contamination from $W Z, Z / W+$ jets, $W W$ and top, and also larger uncertainties associated with the background determination and the missing energy estimation. All measurements performed, by both experiments with 7 and $8 \mathrm{TeV}$ data in the two channels, are in a good agreement with theory [14, 15, 16, 17, 18]. Here, for illustration, the $8 \mathrm{TeV}$ results are given: ATLAS 7.1 $1_{-0.4}^{+0.5}$ (stat.) \pm 0.3 (syst.) \pm 0.2 (lumi.) [17], and CMS 7.7 $7_{-0.5}^{+0.5}$ (stat. $)_{-0.4}^{+0.5}$ (syst.) $\pm 0.4($ th. $) \pm 0.3$ (lumi.) pb [18]. The difference between the two measurements is due to the different definitions of the on-shell mass range. For ATLAS the on-shell region is $66-116 \mathrm{GeV}$, while for $\mathrm{CMS}$ it is $60-120 \mathrm{GeV}$. These measurements should be compared with the predictions $7.2_{-0.2}^{+0.3} \mathrm{pb}$ and $7.7 \pm 0.6 \mathrm{pb}$, respectively. It should be noted, that the $Z Z$ measurement is the only one for which the statistical error is still larger than the systematic error.

$W V$ and $V Z$ Besides the diboson cross section measurements performed using the fully leptonic final states, measurements have been also carried in the semi-leptonic final states. In this case, only one of the two bosons decays in the leptonic final state, and the second one decays in a pair of jets. Two such measurements have been performed. The first one is the WV, where the $\mathrm{W}$ decays in a lepton-neutrino pair, and the $\mathrm{V}$, which is a $\mathrm{Z}$ or a $\mathrm{W}$ boson, decays in two jets. 
The signature is 2 high transverse momentum jets, one prompt lepton, and missing energy. This final state suffers from high background contamination, mainly from $W / Z+$ jets events, and high systematic uncertainties. As the mass resolution of the 2 jets system is poor, one can not distinguish between the $\mathrm{W}$ and the $\mathrm{Z}$ in order to measure separately the $W Z$ and $W W$ processes, and thus, the sum $W W+W Z$ is measured instead. This study has been performed by both experiments with $7 \mathrm{TeV}$ data. CMS finds $68.9 \pm 8.7$ (stat.) \pm 9.7 (syst.) \pm 1.5 (lumi.) $\mathrm{pb}$, to be compared with $65.6 \pm 2.2 \mathrm{pb}$ [19], and ATLAS finds $72 \pm 9$ (stat.) \pm 15 (syst.) \pm 13 (MCstat.) pb to be compared with $63.4 \pm 2.6 \mathrm{pb}$ [20]. Both experiments are in agreement with the NLO MCFM prediction within the large statistical and systematic errors. The second semi-leptonic measurement performed by CMS using the $8 \mathrm{TeV}$ data is the $V Z$ [21], where the $\mathrm{V}$ can be $W \rightarrow l v$ or $Z \rightarrow l l$ or $Z \rightarrow v v$, and the $\mathrm{Z}$ decays always in a $b \bar{b}$ pair. This is also an analysis with high background contamination from $Z b b, W b b, t \bar{t}$ processes, and high systematic uncertainties. In order to discriminate the signal from the background, multivariant techniques have been used. A $2 \mathrm{D}$ fit has been performed in order to extract simultaneously the $W Z$ and the $Z Z$ cross section. This measurement gives a $Z Z$ cross section of $6.5 \pm 1.7$ (stat.) \pm 1.0 (syst.) \pm 0.9 (th. $) \pm 0.2$ (lumi.) pb, which is compatible with the NLO prediction $7.7 \pm 0.4 \mathrm{pb}$, and a $W Z$ cross section of $30.7 \pm 9.3($ stat. $) \pm 7.1$ (syst. $) \pm 4.1($ th. $) \pm$ 1.0 (lumi.) pb, which is also compatible with the prediction $22.3 \pm 1.1 \mathrm{pb}$. This measurement is also dominated by large uncertainties.

\section{Anomalous triple gauge couplings}

The non-abelian standard model structure strictly prescribes the allowed triple gauge boson couplings. At tree level, the charged vector boson couplings are allowed while the neutral are forbidden, and any deviation from this prescription will indicate new physics. The deviations can be parametrised in terms of anomalous couplings using a model independent effective Lagrangian [22]. If new physics exists, it will manifest itself at high boson energies. As a result, in addition to the enhanced cross section, the spectrum of the boson transverse momentum, or the mass of the diboson system will be modified, especially at high values. As the anomalous triple gauge couplings (aTGC) sensitivity depends on the spectrum, limits are usually extracted in bins of transverse boson momentum.

The majority of the diboson analyses, described in the previous section, have also set limits on the anomalous triple gauge couplings. Both experiments have provided limits on all charged and neutral anomalous triple gauge couplings using the $7 \mathrm{TeV}$ data $[3,4,5,8,9,11,14,15,19]$. For the $8 \mathrm{TeV}$ data, only CMS has provided so far limits on the neutral ZZ aTGCs, using the full 2012 dataset $[16,18]$.

Figure 1 summarizes the one-coupling limits. The two top figures present the limits on the charged anomalous triple gauge couplings that have been measured so far by ATLAS and CMS, and these numbers are compared to D0 and LEP limits. The LHC limits are close, and for some coupling parameters event tighter, than the LEP combined limits. The upcoming $8 \mathrm{TeV}$ limits on all coupling parameters are expected to be the best world limits. The two bottom figures, show the one-dimensional limits on the neutral aTGCs. The new CMS $8 \mathrm{TeV}$ limits are about 4 times tighter than the $7 \mathrm{TeV}$ limits. All limits extracted so far are compatible with the SM. It should be 

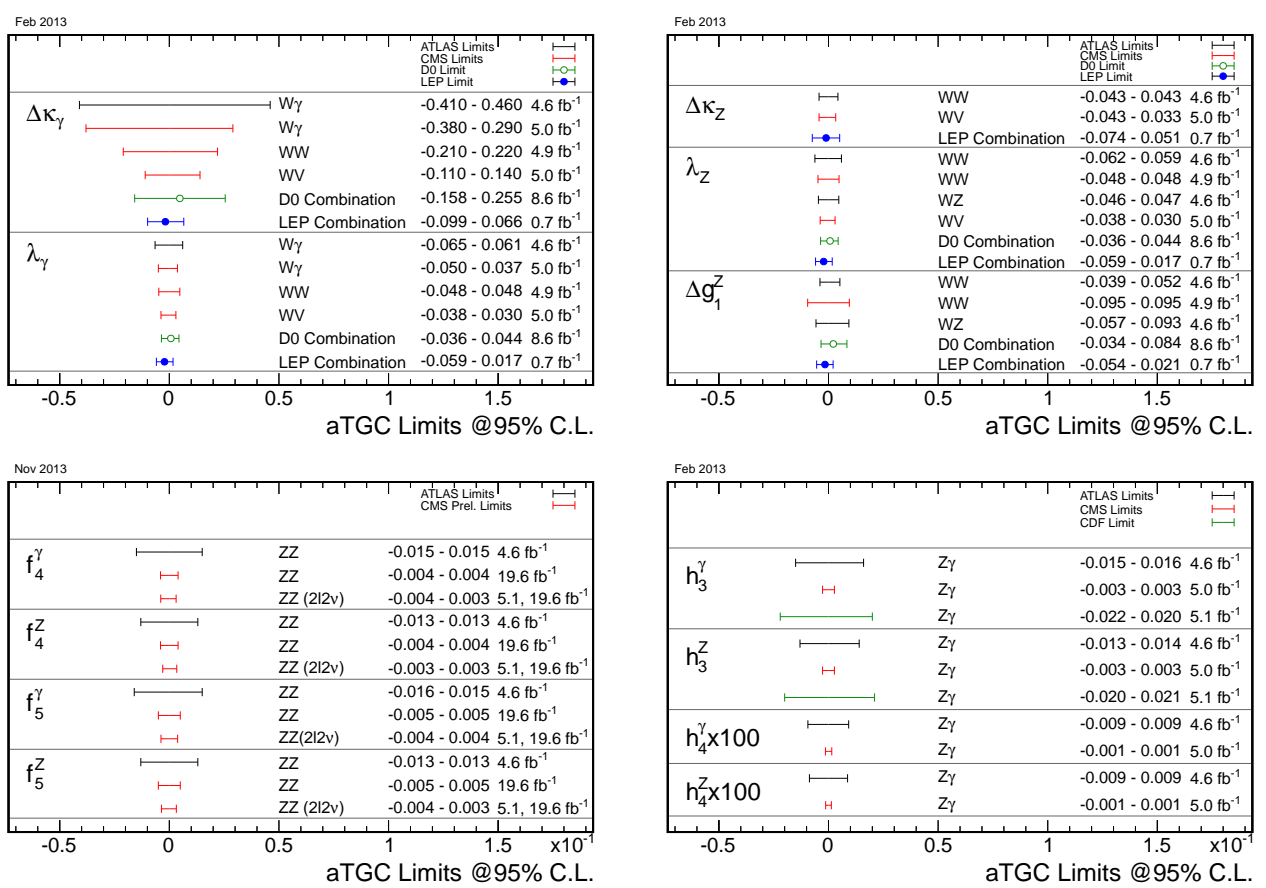

Figure 1: Limits on the charged (top) and neutral (bottom) anomalous triple gauge couplings.

noted that in addition to the one-coupling limits shown here, experiments also provide correlated two coupling limits, which are also compatible with the SM.

\section{Higgs to diboson}

The very clean signature of the $H \rightarrow V V(V=W, Z)$, when the two bosons decay leptonicaly allows a low background analysis. Furthermore, these two diboson decay channels, $W W$ and the $Z Z$, are those with the higher branching ratio. They have been both used for the Higgs discovery, but also for very interesting studies on the Higgs properties (couplings, mass, Spin/Parity measurement and width), which are not covered here.

$H \rightarrow W W \quad$ The $W W$ diboson decay channel is the one with the highest branching ratio, about $22 \%$, and it is probed in the case where both $W$ decay in a lepton-neutrino pair. The highest background contamination to this final state is the irreducible SM $W W$ process. The disadvantage of this final state is that it is not fully reconstructible due to the two neutrinos. Both ATLAS and CMS have published results on the $H \rightarrow W W$ discovery, combining 7 and $8 \mathrm{TeV}$ data. ATLAS measures an excess of events over the background of $3.8 \sigma$ with a signal strength of $1.01 \pm 0.21$ (stat. $) \pm$ 0.19 (th.syst.) \pm 0.12 (exp.syst.) \pm 0.04 (lumi.) [23, 24], while CMS measures an excess of events over the background of $4.3 \sigma$ and a signal strength of $0.72_{-0.12}^{+0.12}(\text { stat. })_{-0.10}^{+0.12}(\text { th.syst. })_{-0.10}^{+0.10}$ (exp.syst) [25].

$H \rightarrow Z Z$ The Higgs decay channel with the greater interest, the so called golden channel, is the $H \rightarrow Z Z \rightarrow l l l l$, as the final state consists of four high energy isolated leptons. Due to the very clean final state, the only important background is the irreducible SM ZZ continuum. The 
disadvantage of this channel is the small branching ratio of the $\mathrm{Z}$ going to a lepton pair in addition to the already small Higgs cross section. In order to increase the available statistics, the analysis strategy is to maximize the lepton acceptance. Combining the 7 and $8 \mathrm{TeV}$ data, ATLAS observes an excess of events over the background of $6.6 \sigma$ with a signal strength of $1.7_{-0.4}^{+0.5}$ [26] and CMS observes a $6.8 \sigma$ signal with a signal strength of $0.93_{-0.23}^{+0.26}(\text { stat. })_{-0.09}^{+0.13}($ syst. $)$ [27].

\section{Conclusion}

All the diboson-related measurements shown in this paper are compatible within uncertainties with the SM predictions. The ratio of the measured cross section over the expected cross section, for all the studies performed with the ATLAS and the CMS detector using 7 and $8 \mathrm{TeV}$ data, are summarized in figures 2. The results are compatible with the SM within uncertainties. The limits on the anomalous triple gauge couplings have been extracted and they confirm that there are no signs of new physics yet. Finally, the Higgs discovery in the diboson, $Z Z$ and $W W$, decay channels has been shown, and the observed signal is compatible, within uncertainties, with a SM Higgs.
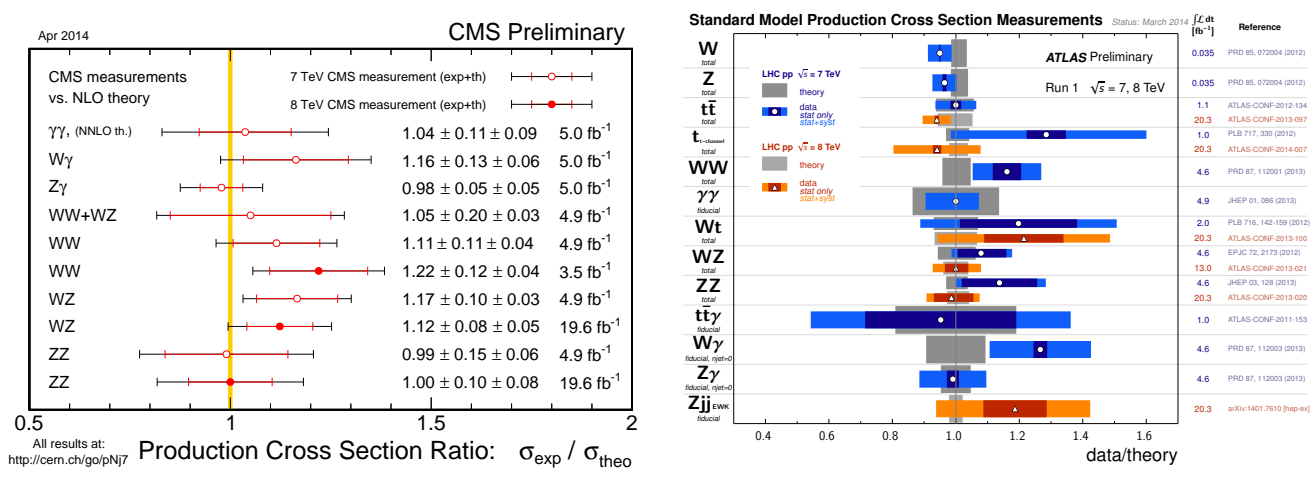

Figure 2: Diboson cross section ratio for CMS (left) and ATLAS (right).

\section{References}

[1] ATLAS Collaboration, 2008 JINST 3 S08003

[2] CMS Collaboration, 2008 JINST 3 S08004

[3] CMS Collaboration, Phys. Rev. D 89 (2014) 092005

[4] ATLAS Collaboration, Phys. Rev. D 87 (2013) 11, 112003

[5] CMS Collaboration, JHEP 1310, 164 (2013)

[6] M. Grazzini et all, http://moriond.in2p3.fr/QCD/2014/ThursdayMorning/Grazzini.pdf

[7] M. Grazzini, S. Kallweit, D. Rathlev and A. Torre, Phys. Lett. B 731 (2014) 204

[8] ATLAS Collaboration, Phys. Rev. D 87 (2013) 11, 112001

[9] CMS Collaboration, Eur. Phys. J. C 73 (2013) 2610

[10] CMS Collaboration, Phys. Lett. B 721 (2013) 190

[11] ATLAS Collaboration, Eur. Phys. J. C 72 (2012) 2173

[12] CMS Collaboration, CMS-PAS-SMP-12-006, https://cds.cern.ch/record/1564318

[13] ATLAS Collaboration, ATLAS-CONF-2013-021. https://cds.cern.ch/record/1525557

[14] CMS Collaboration, JHEP 1301 (2013) 063
[15] ATLAS Collaboration, JHEP 1303 (2013) 128

[16] CMS Collaboration, CMS-PAS-SMP-12-016, https://cds.cern.ch/record/1633371

[17] ATLAS Collaboration, ATLAS-CONF-2013-020, https://cds.cern.ch/record/1525555

[18] CMS Collaboration, submitted to Phys. Lett. B, arXiv:1406.0113

[19] CMS Collaboration, Eur. Phys. J. C 73 (2013) 2283

[20] ATLAS Collaboration, ATLAS-CONF-2012-157, https://cds.cern.ch/record/1493586

[21] CMS Collaboration, CMS-SMP-13-011, https://cds.cern.ch/record/1668410

[22] K. Hagiwara, R. D. Peccei, D. Zeppenfeld and K. Hikasa, Nucl. Phys. B 282 (1987) 253.

[23] ATLAS Collaboration, ATLAS-CONF-2013-030, https://cds.cern.ch/record/1527126

[24] ATLAS Collaboration, Phys. Lett. B 726 (2013) 88

[25] CMS Collaboration, JHEP 1401 (2014) 096

[26] ATLAS Collaboration, ATLAS-CONF-2013-013, https://cds.cern.ch/record/1523699

[27] CMS Collaboration, Phys. Rev. D 89 (2014) 092007 